\title{
Toxicology studies of aqueous-alcohol extracts of Harpagophytum procumbens subsp. procumbens (Burch.) DC.Ex Meisn. (Pedaliaceae) in female and male rats
}

\author{
Kirtan Joshi ${ }^{1^{*}}$, Alan Parrish², Elizabeth A. Grunz-Borgmann², Mary Gerkovich ${ }^{3}$ and William R. Folk ${ }^{1^{*}}$ (D)
}

\begin{abstract}
Background: A variety of medicinal products prepared from secondary tubers of Harpagophytum procumbens subsp. procumbens (Burch.) DC.ex Meisn. (Devil's Claw) and H. zeyheri are marketed in Africa, Europe, the United States, South America and elsewhere, where they are used for inflammatory and musculoskeletal conditions such as arthritis, lower back pain, rheumatism and neuralgia, etc. While clinical studies conducted over the last twenty years support the general safety of such products, infrequent gastrointestinal disturbances (diarrhea, nausea, vomiting, abdominal pain), headache, vertigo and hypersensitivity (allergic) reactions (rash, hives and face swelling) have been documented. Sex-related differences occur in the health conditions for which Devil's Claw products are used, so it is likely that usage is similarly sex-related and so might be side effects and potential toxicities. However toxicologic studies of Devil's Claw products have been conducted primarily with male animals. To address this deficit, we report toxicological studies in female and male rats of several H. procumbens (HP) aqueous-alcohol extracts chemically analyzed by UPLC-MS.

Methods: Female and male Sprague Dawley rats were studied for one and three months in groups differing by consumption of diets without and with HP extracts at a 7-10-fold human equivalent dose (HED). Sera were analyzed for blood chemistry, and heart, liver, lung, kidney, stomach, and small and large intestine tissues were examined for histopathology. Treatment group differences for blood chemistry were analyzed by ANOVA with Dunnett's test and significant group differences for endpoints with marginal distributional properties were verified using the Kruskal-Wallis test. Group differences for histopathology were tested using Chi Square analysis.
\end{abstract}

Results: Significant group by sex-related differences in blood chemistry were detected in both studies. Additionally, several sex-related differences occurred between the studies. However, significant histopathology effects associated with the consumption of the extracts were not detected.

Conclusion: Toxicologic analysis of Devil's Claw extracts cause significant sex-related effects in blood chemistry. However, in our judgement, none of the observed effects suggest serious toxicity at these doses and durations. Subsequent toxicologic and clinical studies of $\mathrm{H}$. procumbens and other medicines with similar properties should explore in greater detail the basis and consequences of potential sex-related effects.

Keywords: Harpagophytum procumbens, Devil's claw, Inflammation, Musculoskeletal, Toxicology, Rats, Sex-related

\footnotetext{
*Correspondence: kvj5kc@health.missouri.edu; folkw@missouri.edu

'Department of Biochemistry, 117 Schweitzer Hall, University of

Missouri-Columbia, Columbia, MO 65211, USA

Full list of author information is available at the end of the article
}

(c) The Author(s). 2020 Open Access This article is distributed under the terms of the Creative Commons Attribution 4.0 International License (http://creativecommons.org/licenses/by/4.0/), which permits unrestricted use, distribution, and reproduction in any medium, provided you give appropriate credit to the original author(s) and the source, provide a link to the Creative Commons license, and indicate if changes were made. The Creative Commons Public Domain Dedication waiver (http://creativecommons.org/publicdomain/zero/1.0/) applies to the data made available in this article, unless otherwise stated. 


\section{Background}

H. procumbens is an important traditional medicinal plant of sub-Sarahan Africa first used by the San and Khoi peoples. It is a weedy, perennial tuberous plant with fruits having numerous long spines with hooks, giving rise to the colloquial name of the genus, Devil's Claw. Secondary tubers are processed into extracts used throughout Africa, Europe and the Americas for treatment of musculoskeletal, inflammatory and other health problems that cause a major source of pain, loss of function and disability for many humans. Individuals with these conditions frequently employ botanicals as primary or supplementary medical treatments, however, scientific evidence of safety and efficacy of most such practices is limited. Devil's Claw products containing $H$. procumbens and/or $H$. zeyheri are widely used to treat inflammatory, immunological and musculoskeletal conditions such as arthritis, lower back pain, rheumatism and neuralgia, as well as other conditions [1-5]. The limited clinical studies include reports of occasional gastrointestinal, kidney and cardiovascular effects but their causes have not been investigated $[1,5]$. Concern about potential toxicities is accentuated by the variety of products, none of which are chemically well defined [1-3]; and that most reported preclinical toxicologic studies have been conducted with male animals, despite evidence that females experience higher inflammatory and immune system responses than males [6-8].

Sex-related differences occur in the incidence of conditions for which Devil's Claw products are used, e.g. osteoarthritis, which has an inflammatory component [9-11] and whose prevalence increases with age, especially when comparing women of post-menopausal age with men of comparable age [8, 10-15]. These findings have led to the hypothesis that decreasing levels of circulating estrogen following menopause are associated with increased production of inflammatory cytokines [8, 1014, 16-19]. Similar sex-related effects may occur with other conditions. Addressing such sex-related differences is important for understanding the safety and effectiveness of Devil's Claw products and the many other medical treatments for inflammatory and immune system conditions. To help redress the lack of sex-related toxicologic data of Devil's Claw products, we conducted one- and three-month studies in female and male adult rats of aqueous-alcoholic $H$. procumbens extracts characterized chemically by UPLC-MS for anti-inflammatory secondary metabolites.

\section{Methods}

\section{Preparation and characterization of extracts}

Dried $H$. procumbens secondary tubers were sourced by Parceval Herbal Products, LLC (Wellington, South Africa) in compliance with the South Africa Biodiversity
Act. Mr. Ulrich Feiter confirmed identity of the plant materials. A botanical voucher (NBG 1488135-0) has been deposited at the Compton Herbarium, South African National Botanical Institute, Kirstenbosch Botanical Garden, Cape Town, South Africa. The dried, milled, plant material was processed as described in U.S. Patent 6280737B1(example 2): Approximately $100 \mathrm{~kg}$ was suspended in $\sim 10 \mathrm{x}$ weight purified water at $85^{\circ} \mathrm{C}$ for two hours, allowed to cool to room temperature and the residue pressed to recover supernatant; the residue was washed with approximately $4 \mathrm{x}$ initial weight of purified water (at $85^{\circ} \mathrm{C}$ ) for two hours and cooled to room temperature, then again pressed and the supernatant recovered. The combined supernatants were filtered through $10 \mu \mathrm{M}$ and $1 \mu \mathrm{M}$ filters and vacuum dried at $50{ }^{\circ} \mathrm{C}$ to approximately $50 \%$ moisture. This 'primary extract' was added slowly to $96 \% \mathrm{v} / \mathrm{v}$ ethanol (approximately $300 \mathrm{~kg}$ ) while stirring, and a precipitate was allowed to form over two hours. The supernatant was carefully removed and concentrated under vacuum. A portion was further treated with water saturated nbutanol as described by U.S. Patent 6280737B1 (example 5). Two independent "primary' aqueous - ethanolic extracts having 5.5\% harpagoside (2014 HP) and 3.3\% harpagoside (2016 HP) were studied as well as the 2016 HP extract further purified with water saturated nbutanol, which resuled in a five-fold enrichment of harpagoside.

All extracts were analyzed by UPLC (with diode detection) - TOF-MS plus MS/MS (with lockspray ionization operated in the ESI negative mode) for candidate bioactive secondary metabolites characteristic of $H$. procumbens [3] (Fig. 1). LC-MS analyses were performed on a Bruker maXis impact quadrupole-time-of-flight mass spectrometer coupled to a Waters ACQUITY UPLC system. Separation was achieved on a Waters C18 column $(2.1 \times 100 \mathrm{~mm}, \mathrm{BEH} \mathrm{C} 18$ column with 1.7 -um particles $)$ using a linear gradient and mobile phase A (0.1\% formic acid) and B (B: acetonitrile). Gradient condition: B increased from 5 to $70 \%$ over $30 \mathrm{~min}$, then to $95 \%$ over 3 min, held at $95 \%$ for $3 \mathrm{~min}$, then returned to $5 \%$ for equilibrium. The flow rate was $0.56 \mathrm{~mL} / \mathrm{min}$ and the column temperature was $60^{\circ} \mathrm{C}$. Mass spectrometry was performed in the negative electrospray ionization mode with the nebulization gas pressure at 43.5 psi, dry gas of $12 \mathrm{~L} / \mathrm{min}$, dry temperature of $250 \mathrm{C}$ and a capillary voltage of $4000 \mathrm{~V}$. Mass spectral data were collected from 100 and $1500 \mathrm{~m} / \mathrm{z}$ and were auto-calibrated using sodium formate after data acquisition. Quantitation of harpagoside concentration was performed using UV at 280 $\mathrm{nm}$. Standard curve was generated using authentic harpagoside standard of 6 different concentrations. Harpagoside in the samples was quantified using the peak area at UV280 nm and the calibration curve. The 


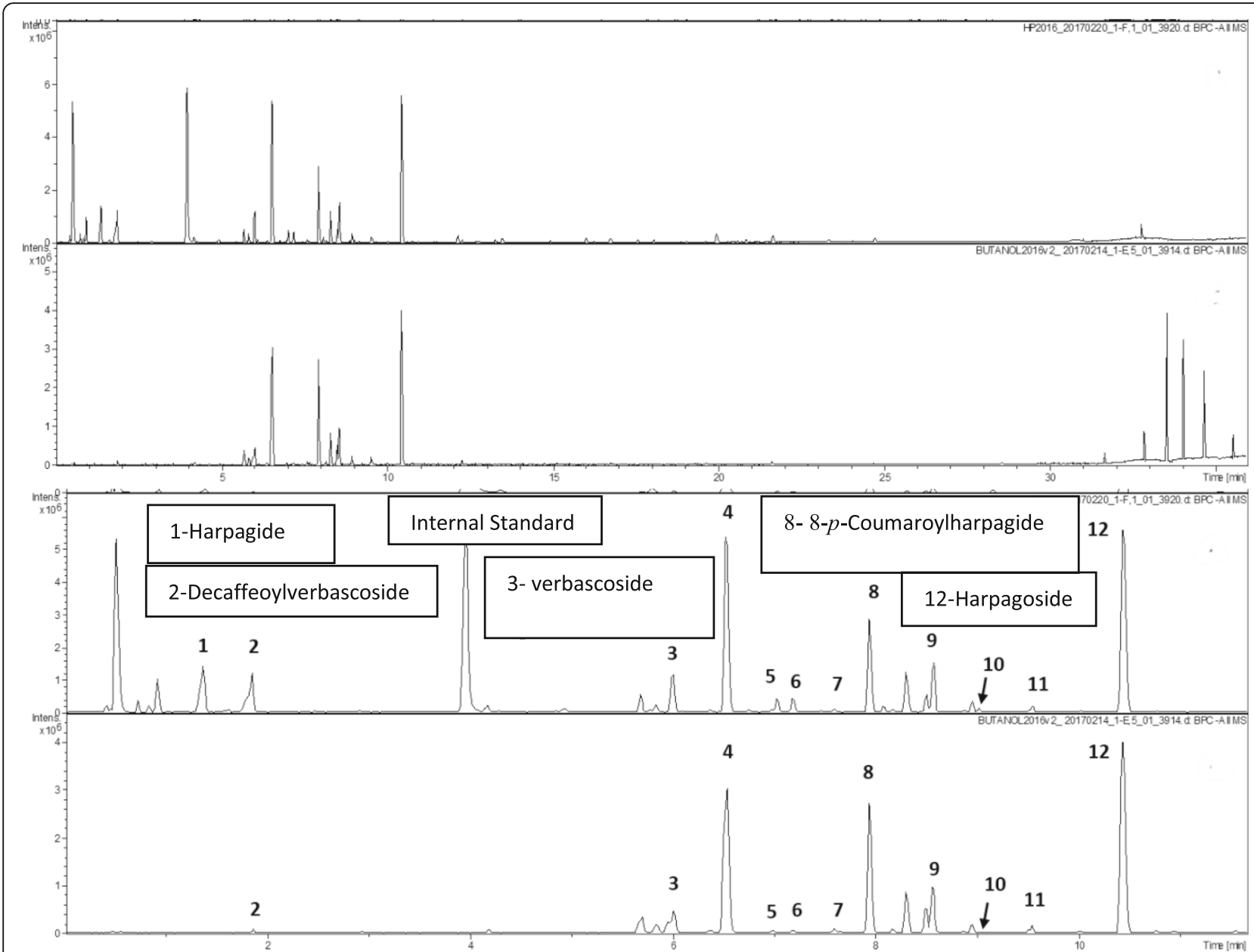

Fig. 1 UPLC-DAD-MS analysis of metabolites in aqueous-ethanolic and butanolic extracts of H. procumbens (top two panels, total elution profile); bottom two panels, portion of the gradient (min. 1-15) in which iridoid and phenylethanoid/phenylpropanoid anti-inflammatory metabolites elute. Marked peaks were identified by comparison with commercial analytical standards

concentrations of the harpagoside was then used calculated the amount of harpagoside in the samples. All extracts fulfill the requirement of European Pharmacopoeia that Devil's Claw products contain no less than $1.2 \%$ harpagoside, and their compositions qualitatively resemble Devil's Claw products marketed in Europe and the U.S. (data not shown).

\section{Study design and treatment}

Adult Sprague Dawley rats were housed in a University of Missouri vivarium under the care of licensed veterinarians with a protocol (\#8654) approved by the University of Missouri Institutional Animal Care and Use Committee. Animals were acclimated for seven days, and 12:12 light and dark cycles were maintained with lights on at 0700 and off at 1900. All animals were monitored twice daily for health status and were singly housed. Animals were euthanized at 0900 with Ketamine/xylazine, 73 and $17 \mathrm{mg} / \mathrm{kg}$ followed terminal heart stick and cervical dislocation or induction of bilateral pneumothoraces as secondary means of assuring euthanasia. There are no deviations from the standards and regulations promulgated under the Animal Welfare Act.

One-month study: Male rats were obtained from Dr. Kathy Timms (University of Missouri) and female rats were purchased from Charles River (Raleigh, USA). Animals randomly assigned to groups of six males and females each were daily fed rodent chow with a peanut butter 'treat' used to deliver the HP extract. Control animals were provided chow and treats without HP extract, while treatment animals consumed chow and treats containing HP extract such that the daily consumption of harpagoside in the extract was approximately $1 \mathrm{~g} / \mathrm{kg} /$ day. (This is between $7.5 \mathrm{x}$ to $10 \mathrm{x}$ the human equivalent dose (HED) reported in most human clinical studies.) Male rats were fasted overnight for chow to induce them to eat the treat, but female rats ate the treat without fasting. Fasted animals were provided food at 0800 to ensure 
complete treat intake. After 30 days, rats were anesthetized, and plasma collected by heart stick; the animals were euthanized and selected organs (heart, liver, kidney, lungs, stomach, large and small intestine) were collected and fixed in $4 \%$ paraformaldehyde for histopathology.

Three-month study: Male rats were obtained from Dr. Jonathan Green (University of Missouri) and females were purchased from Charles River (Raleigh). Males were randomly assigned to two groups of six animals (treat without extract or treat with 2014HP at 10x the HED). Females were randomly assigned to two groups of eight (treat without extract or treat with 2014HP at 10x the HED) and fasted overnight for chow if they did not regularly eat the treats. Fasted animals were provided food at 0800 to ensure complete treat intake. Blood was collected via saphenous vein every month and after 90 days of HP extract consumption rats were anesthetized and plasma collected by heart stick; the animals were euthanized and organs (heart, liver, kidney, lungs, stomach, large and small intestine) were collected and fixed in $4 \%$ paraformaldehyde for histopathology.

\section{Hematology and histopathology analyses}

Sera and tissue sections were analyzed for blood chemistry and histopathology by the University of Missouri Veterinary Medical Diagnostic Laboratory (VMDL). Blood chemistry was measured by Beckman Coulter AU480 Chemistry Analyzer with the Ion Selective Electrode (ISE) measuring method. For calcium measurement Arsenazo, OSR65117, was used. Creatinine reagent OSR6678 was used for creatinine measurement. For Glucose OSR6621 was used. OSR6222 was used for inorganic phosphate measurement. Urea Nitrogen (Bun) reagent OSR6634 was used for urea nitrogen.

The visceral organs including lung, liver, kidney, heart, stomach, small and large intestines were collected and fixed in 10\% neutral-buffered formalin. For histopathological examination by a board-certified veterinary pathologist, the fixed tissues were trimmed, routinely processed, sectioned at $4 \mu \mathrm{m}$, and stained with hematoxylin and eosin. The pathologist was blinded to the definition of each treatment group. Pathologic lesion from each organ were separately scored from 0 to 3 (0: none, 1: mild, 2: moderate, 3: severe).

Group differences for blood chemistry endpoints were analyzed by ANOVA with Dunnett's test; significant group differences for endpoints with marginal distributional properties were verified using the Kruskal-Wallis test. Hemolysis was confirmed with binary logistic regression to predict hemolysis, Wald chi square statistic. For analyses comparing only control versus the 2014 HP group the Dunnett's test was not used. Group differences for histopathology endpoints were tested using Chi Square analysis.

\section{Results}

Throughout and at the end of the studies, all rats appeared alert, lacking porphyrin secretions and moved about their cages freely. Gross examination indicated all mucus membranes were moist and organs were of normal size and color.

\section{One-month study}

No significant differences in histopathology were noted, other than the control animals experienced a higher incidence of heart inflammation (3 rats) than any of the treatment groups ( $0 \%$ of the rats) (Table 1$)$. Blood glucose, urea nitrogen, potassium, anion gap, globulin and phosphate were altered in treatment groups relative to the control group. Sodium was significantly altered between treatment groups (Table 2), suggestive of mild effects upon electrolyte and acid-base balance. There was no significant elevation of liver enzyme levels (alanine transaminase (ALT) or alkaline phosphatase (ALP) diagnostic of hepatotoxicity, but total bilirubin levels increased significantly compared to the controls. Some of this may be due to increased hemolysis in samples during collection.

Significant sex-related differences occurred for the majority of the measures and group by sex interactions were determined to be significant for sodium, potassium, and phosphate levels (Table 3 ). With group by sex interactions, the differences between the groups varied between male or female data analysis. Sodium for females showed no significant group differences, but for males the control group differed significantly from the 2016 HP and Butanolic HP groups. For potassium, females differed between $2016 \mathrm{HP}$ and the control group. The male control group differed significantly from each of the HP groups. Females in the 2016 HP and Butanolic HP groups exhibited increased phosphate compared to control, while males did not (Table 4). When considering just the sex and group main effects independently, the bicarbonate level elevation in males is indicative of acidosis and may explain the anion gap differences between the control and the treatment group. Additional analysis of other outcome measures is provided in Additional file 1.

We also analyzed 2014 HP and control data from the 1 -month study separately to directly compare it with the 3-month study: Out of 20 variables tested (Table 6) in the blood chemistry panel there were a total of 5 group, 11 sex-related and 3 group by sex-related effects in the 1-month study. (Potassium, CK and bicarbonate having group by sex effects).

\section{Three-month study}

The 3-month study was performed with the 2014 HP extract only; therefore, all statistical comparisons of the 3- 
Table 1 One Month Study Histopathology Analysis: Test of Group Differences ( $n=46 ; 22$ females, 24 males)

\begin{tabular}{|c|c|c|c|c|c|c|}
\hline & & $\begin{array}{l}\text { Control } \\
(n=12)\end{array}$ & $\begin{array}{l}2014 \mathrm{HP} \\
(n=11)\end{array}$ & $\begin{array}{l}2016 \mathrm{HP} \\
(n=11)\end{array}$ & $\begin{array}{l}2016 \text { Butanolic HP } \\
(n=12)\end{array}$ & $X^{2}(d f) p$ \\
\hline & Scoring: & $\begin{array}{l}\text { None - \# (\%) } \\
\text { Mild - \# (\%) }\end{array}$ & $\begin{array}{l}\text { None - \# (\%) } \\
\text { Mild - \# (\%) }\end{array}$ & $\begin{array}{l}\text { None - \# (\%) } \\
\text { Mild - \# (\%) }\end{array}$ & $\begin{array}{l}\text { None - \# (\%) } \\
\text { Mild - \# (\%) }\end{array}$ & \\
\hline \multirow[t]{2}{*}{ Lung } & Inflammation & $\begin{array}{l}12(100) \\
0(0)\end{array}$ & $\begin{array}{l}11(100) \\
0(0)\end{array}$ & $\begin{array}{l}11(100) \\
0(0)\end{array}$ & $\begin{array}{l}12(100) \\
0(0)\end{array}$ & * \\
\hline & Edema & $\begin{array}{l}12(100) \\
0(0)\end{array}$ & $\begin{array}{l}11(100) \\
0(0)\end{array}$ & $\begin{array}{l}11(100) \\
0(0)\end{array}$ & $\begin{array}{l}12(100) \\
0(0)\end{array}$ & * \\
\hline \multirow[t]{5}{*}{ Liver } & Degeneration & $\begin{array}{l}12(100) \\
0(0)\end{array}$ & $\begin{array}{l}11(100) \\
0(0)\end{array}$ & $\begin{array}{l}11(100) \\
0(0)\end{array}$ & $\begin{array}{l}12(100) \\
0(0)\end{array}$ & * \\
\hline & Inflammation & $\begin{array}{l}12(100) \\
0(0)\end{array}$ & $\begin{array}{l}11(100) \\
0(0)\end{array}$ & $\begin{array}{l}11(100) \\
0(0)\end{array}$ & $\begin{array}{l}12(100) \\
0(0)\end{array}$ & * \\
\hline & Necrosis \& hemorrhage & $\begin{array}{l}12(100) \\
0(0)\end{array}$ & $\begin{array}{l}10(91) \\
1(9)\end{array}$ & $\begin{array}{l}11(100) \\
0(0)\end{array}$ & $\begin{array}{l}11(92) \\
1(8)\end{array}$ & 2.10 (3) .55 \\
\hline & Fibrosis & $\begin{array}{l}12(100) \\
0(0)\end{array}$ & $\begin{array}{l}11(100) \\
0(0)\end{array}$ & $\begin{array}{l}11(100) \\
0(0)\end{array}$ & $\begin{array}{l}12(100) \\
0(0)\end{array}$ & * \\
\hline & Apoptosis & $* *$ & $\begin{array}{l}* * \\
1\end{array}$ & $\begin{array}{l}* * \\
1\end{array}$ & $* *$ & $* *$ \\
\hline \multirow[t]{4}{*}{ Kidney } & Glomerulonephritis & $\begin{array}{l}12(100) \\
0(0)\end{array}$ & $\begin{array}{l}11(100) \\
0(0)\end{array}$ & $\begin{array}{l}11(100) \\
0(0)\end{array}$ & $\begin{array}{l}12(100) \\
0(0)\end{array}$ & * \\
\hline & Tubular degeneration / necrosis & $\begin{array}{l}12(100) \\
0(0)\end{array}$ & $\begin{array}{l}9(82) \\
2(18)\end{array}$ & $\begin{array}{l}11(100) \\
0(0)\end{array}$ & $\begin{array}{l}11(92) \\
1(8)\end{array}$ & 4.12 (3) .25 \\
\hline & Interstitial nephritis & $\begin{array}{l}12(100) \\
0(0)\end{array}$ & $\begin{array}{l}10(91) \\
1(9)\end{array}$ & $\begin{array}{l}11(100) \\
0(0)\end{array}$ & $\begin{array}{l}11(92) \\
1(8)\end{array}$ & 2.10 (3) .55 \\
\hline & Proteinuria & $\begin{array}{l}10(83) \\
2(17)\end{array}$ & $\begin{array}{l}6(55) \\
5(45)\end{array}$ & $\begin{array}{l}8(73) \\
3(27)\end{array}$ & $\begin{array}{l}7(58) \\
5(42)\end{array}$ & ${ }_{* * *}^{2.80}(3) .42$ \\
\hline \multirow[t]{3}{*}{ Heart } & Inflammation & $\begin{array}{l}9(75) \\
3(25)\end{array}$ & $\begin{array}{l}11(100) \\
0(0)\end{array}$ & $\begin{array}{l}11(100) \\
0(0)\end{array}$ & $\begin{array}{l}12(100) \\
0(0)\end{array}$ & 9.09 (3) .03 \\
\hline & Necrosis & $\begin{array}{l}12(100) \\
0(0)\end{array}$ & $\begin{array}{l}11(100) \\
0(0)\end{array}$ & $\begin{array}{l}11(100) \\
0(0)\end{array}$ & $\begin{array}{l}12(100) \\
0(0)\end{array}$ & * \\
\hline & Fibrosis & $\begin{array}{l}12(100) \\
0(0)\end{array}$ & $\begin{array}{l}11(100) \\
0(0)\end{array}$ & $\begin{array}{l}11(100) \\
0(0)\end{array}$ & $\begin{array}{l}12(100) \\
0(0)\end{array}$ & * \\
\hline \multirow[t]{2}{*}{ Stomach } & Inflammation & $\begin{array}{l}12(100) \\
0(0)\end{array}$ & $\begin{array}{l}11(100) \\
0(0)\end{array}$ & $\begin{array}{l}11(100) \\
0(0)\end{array}$ & $\begin{array}{l}12(100) \\
0(0)\end{array}$ & * \\
\hline & Mucosal erosion / ulceration & $\begin{array}{l}12(100) \\
0(0)\end{array}$ & $\begin{array}{l}11(100) \\
0(0)\end{array}$ & $\begin{array}{l}11(100) \\
0(0)\end{array}$ & $\begin{array}{l}12(100) \\
0(0)\end{array}$ & * \\
\hline \multirow[t]{2}{*}{ Small Intestine } & Inflammation & $\begin{array}{l}12(100) \\
0(0)\end{array}$ & $\begin{array}{l}11(100) \\
0(0)\end{array}$ & $\begin{array}{l}11(100) \\
0(0)\end{array}$ & $\begin{array}{l}12(100) \\
0(0)\end{array}$ & * \\
\hline & Mucosal erosion / ulceration & $\begin{array}{l}12(100) \\
0(0)\end{array}$ & $\begin{array}{l}11(100) \\
0(0)\end{array}$ & $\begin{array}{l}11(100) \\
0(0)\end{array}$ & $\begin{array}{l}12(100) \\
0(0)\end{array}$ & * \\
\hline \multirow[t]{2}{*}{ Large Intestine } & Inflammation & $\begin{array}{l}12(100) \\
0(0)\end{array}$ & $\begin{array}{l}11(100) \\
0(0)\end{array}$ & $\begin{array}{l}11(100) \\
0(0)\end{array}$ & $\begin{array}{l}12(100) \\
0(0)\end{array}$ & * \\
\hline & Mucosal erosion / ulceration & $\begin{array}{l}12(100) \\
0(0)\end{array}$ & $\begin{array}{l}11(100) \\
0(0)\end{array}$ & $\begin{array}{l}11(100) \\
0(0)\end{array}$ & $\begin{array}{l}12(100) \\
0(0)\end{array}$ & * \\
\hline
\end{tabular}

\# Data included in the attached Excel file

* Chi-square test statistic of differences in level of response between groups not computed due to distribution of responses - no variability in level of response ** Liver apoptosis was not recorded for any male animals; only noted as "mild" in 2 female rats; no coding for the other female rats and all the male rats

${ }^{* * *}$ An additional statistical test comparing control and 2014 HP groups. X2 (df) p $\rightarrow 2.25$ (1) .13. No significant difference between the two most extreme groups

month study and 1-month study used only data for the 2014 HP extract and relevant control groups. Since the studies were performed at different times, the statistical data is presented separately and differences between the two studies noted.

As with the 1-month study, histopathology analysis found no significant effects for any of the outcome variables tested. Kidney interstitial nephritis, proteinuria and tubular degeneration/necrosis were noted, however, none of these were statistically significant (Additional file 1:). Out of 20 variables tested in the blood chemistry panel there were 1 group, 12 sex-related and 2 group by sex-related effects. Phosphorus and bilirubin were the only variables with a group by sex-related effect. All the 
Table 2 One Month Study Blood Chemistry Group ANOVA

\begin{tabular}{|c|c|c|c|c|c|}
\hline \multirow[b]{2}{*}{ Measure } & \multirow[b]{2}{*}{$\begin{array}{l}F, p \text { value } \\
\text { (df } 3,42)\end{array}$} & \multicolumn{4}{|l|}{ Mean (sd) } \\
\hline & & Control & $2014 \mathrm{HP}$ & 2016 HP & $\begin{array}{l}2016 \\
\text { Butanolic } \\
\text { HP }\end{array}$ \\
\hline Glucose (mg/dL) & $3.38, .03$ & $\begin{array}{l}297.92 \\
(61.40)\end{array}$ & $\begin{array}{l}248.27 \\
(43.67) * \\
(p=.048)\end{array}$ & $\begin{array}{l}239.82 \\
(34.52) * \\
(p=.02)\end{array}$ & $\begin{array}{l}253.42 \\
(48.58)\end{array}$ \\
\hline Urea Nitrogen (mg/dL) & $15.50,<.001$ & $\begin{array}{l}20.67 \\
(3.45)\end{array}$ & $20.82(2.27)$ & $\begin{array}{l}14.82(1.72) \\
*(p<.001)\end{array}$ & $\begin{array}{l}19.00 \\
(1.48)\end{array}$ \\
\hline Creatinine $(\mathrm{mg} / \mathrm{dL})^{* * *}$ & $1.33, .28$ & & & & \\
\hline Sodium (mEq/L) & $3.28, .03$ & $\begin{array}{l}142.00 \\
(2.37)\end{array}$ & $\begin{array}{l}143.18(1.78) \\
* *(p=.017)\end{array}$ & $\begin{array}{l}140.36(1.69) \\
* *(p=.017)\end{array}$ & $\begin{array}{l}141.75 \\
(2.45)\end{array}$ \\
\hline Potassium (mEq/L) & $11.83,<.001$ & $4.82(0.37)$ & $5.66(0.86)$ & $\begin{array}{l}6.95(0.65) * \\
(p<.001)\end{array}$ & $\begin{array}{l}6.53(1.47) \\
*(p<.001)\end{array}$ \\
\hline Chloride (mEq/L) & $1.34, .27$ & & & & \\
\hline Bicarbonate (mEq/L) & $1.87, .15$ & & & & \\
\hline Anion Gap (mEq/L) & $4.42, .009$ & $\begin{array}{l}18.00 \\
(1.54)\end{array}$ & $\begin{array}{l}20.46(1.13) * \\
(p=.003)\end{array}$ & $\begin{array}{l}19.73(1.79) \\
*(p=.049)\end{array}$ & $\begin{array}{l}19.67 \\
(2.10) \\
(p=.052)\end{array}$ \\
\hline Albumin (g/dL) & $0.09, .97$ & & & & \\
\hline Total Protein (g/dL) & $0.63, .60$ & & & & \\
\hline Globulin (g/dL) & $3.53, .023$ & $2.77(0.22)$ & $2.58(0.20)$ & $\begin{array}{l}2.52(0.19) * \\
(p=.009)\end{array}$ & $2.62(0.15)$ \\
\hline Calcium (mg/dL) & $0.06, .98$ & & & & \\
\hline Phosphorus (mg/dL) & $3.89, .015$ & $7.04(0.65)$ & $\begin{array}{l}7.74(0.66) * \\
(p=.035)\end{array}$ & $\begin{array}{l}7.87(0.60) * \\
(p=.009)\end{array}$ & $7.68(0.66)$ \\
\hline Cholesterol (mg/dL) & $0.06, .98$ & & & & \\
\hline Total Bilirubin $(\mathrm{mg} / \mathrm{dL})^{* * *}$ & $25.08,<.001$ & $0.10(0.00)$ & $\begin{array}{l}0.21(0.05) * \\
(p<.001)\end{array}$ & $\begin{array}{l}0.23(0.05) * \\
(p<.001)\end{array}$ & $\begin{array}{l}0.23(0.05) \\
*(p<.001)\end{array}$ \\
\hline $\mathrm{ALT}(\mathrm{U} / \mathrm{L})$ & $1.29, .29$ & & & & \\
\hline $\mathrm{ALP}(\mathrm{U} / \mathrm{L})$ & $1.76, .17$ & & & & \\
\hline GGT (U/L) & \multicolumn{5}{|c|}{ All values were " $<3$ " - no analyses conducted } \\
\hline \multirow[t]{2}{*}{ CK $(U / L) * * * *$} & $2.75, .06$ & $\begin{array}{l}334.58 \\
(143.97)\end{array}$ & $\begin{array}{l}467.55 \\
(277.07)\end{array}$ & $\begin{array}{l}246.73 \\
(114.43)\end{array}$ & $\begin{array}{l}242.42 \\
(265.53)\end{array}$ \\
\hline & $\begin{array}{l}x^{2}, p \text { value } \\
(d f 3)\end{array}$ & $\begin{array}{l}\text { Control } \\
\% \text { Slight } \\
\text { Hemolysis }\end{array}$ & $\begin{array}{l}2014 \text { HP } \\
\% \text { Slight } \\
\text { Hemolysis }\end{array}$ & $\begin{array}{l}2016 \text { HP } \\
\% \text { Slight } \\
\text { Hemolysis }\end{array}$ & $\begin{array}{l}\text { Butanolic } \\
\text { HP } \\
\% \text { Slight } \\
\text { Hemolysis }\end{array}$ \\
\hline $\begin{array}{l}\text { Hemolysis (Mild to moderate hemolysis may cause false increases in direct } \\
\text { bilirubin and AST measurement. Marked hemolysis can adversely affect all } \\
\text { chemistry tests.) }\end{array}$ & $0.32, .96$ & $25 \%$ & $18 \%$ & $18 \%$ & $17 \%$ \\
\hline
\end{tabular}

\footnotetext{
* Intervention group(s) was significantly $(p<.05)$ different from control group

** Intervention groups were significantly different from each other

***Based on distributional properties of the outcome measure, group differences were also tested using nonparametric Kruskal-Wallis Test

Creatinine $\mathrm{X} 2=4.04, \mathrm{df} 3, p=.257$

Total Bilirubin X2 $=30.34$, df $3, p<.001$

${ }_{* * * *}$ Note - when outlier value (Data_id $=34$ ) was excluded from the analysis, $F(3,41)=1.81, p=.16$
}

significant interactions are listed in bold in Table 5 (3month).

While there were many sex related effects observed in the blood chemistry panel, bilirubin was the only variable that had a significant difference between the control and treatment groups in both 1-month and 3-month time points.

\section{Discussion}

A variety of Devil's Claw products and dietary supplements have been commercially marketed for over 50 years and are widely used for inflammatory health conditions such as arthritis, lower back pain, rheumatism and neuralgia [20]. While clinical studies conducted over the past twenty years support the general safety of such 
Table 3 One Month Study Blood Chemistry Group by Sex ANOVA

\begin{tabular}{|c|c|c|c|}
\hline Measure & $\begin{array}{l}\text { GROUP } \\
\text { main effect } \\
F, p \text { value } \\
(\text { df } 3,38)\end{array}$ & $\begin{array}{l}\text { SEX main } \\
\text { effect } \\
F, p \text { value (df } \\
1,38)\end{array}$ & $\begin{array}{l}\text { GROUP X } \\
\text { SEX } \\
\text { interaction } \\
F, p \text { value } \\
(\text { df } 3,38)\end{array}$ \\
\hline Glucose (mg/dL) & $4.97, .005$ & $17.50,<.001$ & $1.05, .38$ \\
\hline Urea Nitrogen (mg/dL) & $16.15,<.001$ & $0.06, .81$ & $1.43, .25$ \\
\hline Creatinine (mg/dL) & $1.91, .15$ & $30.79,<.001$ & $1.22, .31$ \\
\hline Sodium (mEq/L) & $4.54, .01$ & $8.97, .005$ & $4.47, .01 *$ \\
\hline Potassium (mEq/L) & $78.88,<.001$ & $154.09,<.001$ & $\begin{array}{l}31.05 \\
<.001 *\end{array}$ \\
\hline Chloride (mEq/L) & $1.83, .16$ & $21.08,<.001$ & $0.03, .99$ \\
\hline Bicarbonate (mEq/L) & $3.78, .02$ & $33.07,<.001$ & $2.36, .09$ \\
\hline Anion Gap (mEq/L) & $4.77, .01$ & $2.11, .15$ & $1.99, .13$ \\
\hline Albumin (g/dL) & $0.27, .85$ & $190.31,<.001$ & $0.38, .77$ \\
\hline Total Protein (g/dL) & $1.81, .16$ & $125.86,<.001$ & $1.32, .28$ \\
\hline Globulin (g/dL) & $4.42, .01$ & $13.13, .001$ & $1.90, .15$ \\
\hline Calcium (mg/dL) & $0.14, .94$ & $69.77,<.001$ & $0.24, .87$ \\
\hline Phosphorus (mg/dL) & $5.00, .005$ & $2.31, .14$ & $4.24, .01 *$ \\
\hline Cholesterol (mg/dL) & $0.03, .99$ & $24.10,<.001$ & $0.35, .79$ \\
\hline Total Bilirubin (mg/dL) & $33.00,<.001$ & $10.81, .002$ & $1.95, .14$ \\
\hline $\operatorname{ALT}(U / L)$ & $2.98, .04$ & $45.48,<.001$ & $0.74, .53$ \\
\hline $\operatorname{ALP}(\mathrm{U} / \mathrm{L})$ & $1.86, .15$ & $2.04, .16$ & $2.61, .07$ \\
\hline GGT (U/L) & \multicolumn{3}{|c|}{$\begin{array}{l}\text { All values were " }<3 \text { " - no analyses } \\
\text { conducted }\end{array}$} \\
\hline \multirow[t]{2}{*}{ CK (U/L) } & $3.70, .02$ & $3.79, .06$ & $2.80, .053$ \\
\hline & \multicolumn{3}{|c|}{$\begin{array}{l}\text { Overall Model Test }-X^{2}=9.21, \mathrm{df} 7, \\
p=.24 \\
\text { Binary logistic regression to predict } \\
\text { hemolysis. Wald } X^{2} \text { statistic }\end{array}$} \\
\hline $\begin{array}{l}\text { Hemolysis (Mild to moderate } \\
\text { hemolysis may cause false } \\
\text { increases in direct bilirubin and } \\
\text { AST measurement. Marked } \\
\text { hemolysis can adversely affect } \\
\text { all chemistry tests.) }\end{array}$ & $\begin{array}{l}x^{2}=1.90 \\
\text { df } 3, p=.59\end{array}$ & $\begin{array}{l}X^{2}=0.0, d f \\
1, p=.99\end{array}$ & $\begin{array}{l}X^{2}=0.1, \mathrm{df} \\
3, p=1.0\end{array}$ \\
\hline
\end{tabular}

*Only Sodium, Potassium and Phosphorus are significant for group by sex interactions

products, infrequent gastrointestinal disturbances (diarrhea, nausea, vomiting, abdominal pain), headache and vertigo, electrolyte imbalance, hypertension, and hypersensitivity (allergic) reactions (rash, hives and face swelling) have been documented [4, 21-25].

Most toxicologic studies of Devil's Claw materials have used products that were not chemically defined [26-28] and used only male rats. Two recent studies (also with not well-defined Devil's Claw product) used male and female mice; in these, acute $(24 \mathrm{~h})$ and chronic ( 3 months) treatments caused no significant toxicologic effects [29]. However, mild degenerative changes and fibrin accumulation in lung bronchioles and alveoli were observed as well as
Table 4 One Month Study Blood Chemistry Group ANOVA Tests, separately by Sex

\begin{tabular}{|c|c|c|c|c|}
\hline \multicolumn{5}{|l|}{ Sodium } \\
\hline \multicolumn{5}{|c|}{ Females $F=2.13 ;$ df 3,$18 ; p=.13$} \\
\hline \multicolumn{5}{|c|}{ Males $F=11.79$, df 3,$20 ; p<.001$} \\
\hline \multirow[t]{2}{*}{ Measure } & \multicolumn{4}{|c|}{ Mean (sd) } \\
\hline & Control & $2014 \mathrm{HP}$ & 2016 HP & $\begin{array}{l}2016 \\
\text { Butanolic HP }\end{array}$ \\
\hline $\begin{array}{l}\text { Female - Sodium } \\
(\mathrm{mEq} / \mathrm{L})\end{array}$ & $\begin{array}{l}141.33 \\
(3.08)\end{array}$ & $\begin{array}{l}144.00 \\
(1.58)\end{array}$ & $\begin{array}{l}141.60 \\
(1.52)\end{array}$ & $143.67(2.07)$ \\
\hline $\begin{array}{l}\text { Male - Sodium } \\
(\mathrm{mEq} / \mathrm{L})\end{array}$ & $\begin{array}{l}142.67 \\
(1.37)\end{array}$ & $\begin{array}{l}142.50 \\
(1.76)\end{array}$ & $\begin{array}{l}139.33 \\
(1.03)^{* *}\end{array}$ & $\begin{array}{l}139.83 \\
(0.41)^{* *}\end{array}$ \\
\hline \multicolumn{5}{|l|}{ Potassium } \\
\hline \multicolumn{5}{|c|}{ Females $F=15.69 ;$ df 3,$18 ; p<.001$} \\
\hline \multicolumn{5}{|c|}{ Males $F=125.07$, df 3, 20; $p<.001$} \\
\hline \multirow[t]{2}{*}{ Measure } & \multicolumn{4}{|c|}{ Mean (sd) } \\
\hline & Control & 2014 HP & 2016 HP & $\begin{array}{l}2016 \\
\text { Butanolic HP }\end{array}$ \\
\hline $\begin{array}{l}\text { Female - Potassium } \\
(\mathrm{mEq} / \mathrm{L})\end{array}$ & $\begin{array}{l}4.85 \\
(0.44)\end{array}$ & $\begin{array}{l}4.82 \\
(0.33)\end{array}$ & $\begin{array}{l}6.36 \\
(0.35) * *\end{array}$ & $5.17(0.48)$ \\
\hline $\begin{array}{l}\text { Male - Potassium } \\
(\mathrm{mEq} / \mathrm{L})\end{array}$ & $\begin{array}{l}4.78 \\
(0.32)\end{array}$ & $\begin{array}{l}6.37 \\
(0.29) * *\end{array}$ & $\begin{array}{l}7.43 \\
(0.34) * *\end{array}$ & $7.90(0.24) * *$ \\
\hline \multicolumn{5}{|l|}{ Phosphorus } \\
\hline \multicolumn{5}{|c|}{ Females $F=5.40 ; \mathrm{df} 3,18 ; p=.01$} \\
\hline \multicolumn{5}{|c|}{ Males $F=1.68$, df 3,$20 ; p=.20$} \\
\hline \multirow[t]{2}{*}{ Measure } & \multicolumn{4}{|c|}{ Mean (sd) } \\
\hline & Control & 2014 HP & 2016 HP & $\begin{array}{l}2016 \\
\text { Butanolic HP }\end{array}$ \\
\hline $\begin{array}{l}\text { Female - Phosphorus } \\
(\mathrm{mEq} / \mathrm{L})\end{array}$ & $\begin{array}{l}6.55 \\
(0.50)\end{array}$ & $\begin{array}{l}7.38 \\
(0.65)\end{array}$ & $\begin{array}{l}8.18 \\
(0.64) * *\end{array}$ & $7.70(0.92) * *$ \\
\hline $\begin{array}{l}\text { Male - Phosphorus } \\
(\mathrm{mEq} / \mathrm{L})\end{array}$ & $\begin{array}{l}7.53 \\
(0.31)\end{array}$ & $\begin{array}{l}8.03 \\
(0.55)\end{array}$ & $\begin{array}{l}7.62 \\
(0.45)\end{array}$ & $7.65(0.32)$ \\
\hline
\end{tabular}

**HP group(s) was significantly $(p<.05)$ different from Control Group

degeneration of hepatocytes with accumulated tissue debris in embryos - indicative of liver lesions; and necrosis with lymphocytic perivascular cuffing were observed in the adults. In the embryonic kidney sections, degeneration of proximal and distal convoluted tubules with tissue debris collection in the lumens were observed. In pregnant mice of the same group, necrosis of convoluted tubules and infiltration of mononuclear inflammatory cells along with lymphocytic perivascular cuffing was seen [30]. In none of these studies were sex-related effects evaluated or reported.

In the studies reported here, multiple sex-related effects were observed: Creatine kinase (CK) was differentially altered between the two different study groups and sexes in the 1-month study; in the 3-month study there was no difference between treatment and control group, but a sex- 
Table 5 Three Month Study Blood Chemistry Males and Females Group by Sex ANOVA (bold - significant differences)

\begin{tabular}{|c|c|c|c|c|}
\hline Serum measure & $\begin{array}{l}\text { GROUP } \\
\text { main } \\
\text { effect }\end{array}$ & $\begin{array}{l}\text { SEX } \\
\text { main } \\
\text { effect }\end{array}$ & $\begin{array}{l}\text { GROUP X } \\
\text { SEX } \\
\text { interaction }\end{array}$ & Special Notes *Report as $p<.001$ \\
\hline & $\begin{array}{l}F, p \\
\text { value } \\
(d f 1,24)\end{array}$ & $\begin{array}{l}\text { F, } p \\
\text { value } \\
\text { (df } 1 \\
24)\end{array}$ & $\begin{array}{l}F, p \text { value } \\
(\mathrm{df} 1,24)\end{array}$ & \\
\hline Glucose (mg/dL) & $2.24, .15$ & $\begin{array}{l}18.31 \\
<.001\end{array}$ & $0.56, .46$ & Females $<$ Males \\
\hline Urea Nitrogen (mg/dL) & $3.72, .07$ & $0.28, .60$ & $0.03, .86$ & No significant interaction \\
\hline Creatinine (mg/dL) & $\begin{array}{l}9.10 \\
.006\end{array}$ & $\begin{array}{l}14.41 \\
.001\end{array}$ & $0.01, .92$ & Placebo < 2014 HP \& Females > Males \\
\hline Sodium (mEq/L) & $0.07, .80$ & $4.79, .04$ & $0.93, .35$ & Females > Males \\
\hline Potassium (mEq/L) (> 10 values coded as missing data) & $\begin{array}{l}0.003 \\
.96\end{array}$ & $\begin{array}{l}74.43 \\
<.001\end{array}$ & $0.46, .50$ & Females $<$ Males \\
\hline Chloride (mEq/L) & $0.13, .73$ & $\begin{array}{l}11.99 \\
.002\end{array}$ & $1.94, .18$ & Females $<$ Males \\
\hline Bicarbonate (mEq/L) & $1.22, .28$ & $\begin{array}{l}45.86 \\
<.001\end{array}$ & $0.08, .79$ & Females $>$ Males \\
\hline $\begin{array}{l}\text { Anion Gap (mEq/L) (missing data coded with group } \\
\text { average) }\end{array}$ & $3.12, .09$ & $\begin{array}{l}12.49 \\
.002\end{array}$ & $3.12, .09$ & Females $<$ Males \\
\hline Albumin (g/dL) & $1.93, .18$ & $\begin{array}{l}98.42 \\
<.001\end{array}$ & $0.52, .48$ & Females $>$ Males \\
\hline Total Protein (g/dL) & $\begin{array}{l}4.24 \\
.051\end{array}$ & $\begin{array}{l}55.26 \\
<.001\end{array}$ & $0.46, .50$ & Females $>$ Males \\
\hline Globulin (g/dL) & $2.79, .11$ & $0.06, .80$ & $0.11, .74$ & No significant interaction \\
\hline Calcium (mg/dL) & $0.00, .99$ & $\begin{array}{l}67.10 \\
<.001\end{array}$ & $0.01, .92$ & Females $>$ Males \\
\hline Phosphorus (mg/dL) & $0.26, .61$ & $1.70, .21$ & $4.81, .04$ & $\begin{array}{l}\text { Differences between groups for females and males. } \\
\text { Females: Placebo > } 2014 \text { HP } \\
\text { Males: Placebo < } 2014 \text { HP }\end{array}$ \\
\hline Cholesterol (mg/dL) (outlier coded with group average) & $2.55, .12$ & $\begin{array}{l}42.63 \\
<.001\end{array}$ & $2.95, .10$ & Females $<$ Males \\
\hline Total Bilirubin (mg/dL) & $\begin{array}{l}17.62 \\
<.001\end{array}$ & $6.51, .02$ & $4.94, .04$ & $\begin{array}{l}\text { Significant main effects and interaction. Significant } \\
\text { differences between females and males. For both } \\
\text { females and males, Placebo < } 2014 \text { HP. This difference } \\
\text { was much greater for females than it was for males. } \\
\text { (With a significant interaction, do not interpret either } \\
\text { significant main effect.) }\end{array}$ \\
\hline $\operatorname{ALT}(U / L)$ & $0.86, .36$ & $2.75, .11$ & $1.16, .29$ & No significant interaction \\
\hline ALP (U/L) & $1.38, .25$ & $0.80, .38$ & $1.69, .21$ & No significant interaction \\
\hline GGT (U/L) & \multicolumn{3}{|c|}{$\begin{array}{l}\text { All values were " }<3 \text { " - no } \\
\text { analyses conducted }\end{array}$} & \\
\hline CK (U/L) (outlier value coded with group average) & $0.34, .57$ & $5.60, .03$ & $0.47, .50$ & Females $>$ Males \\
\hline \multirow[t]{2}{*}{$\begin{array}{l}\text { Hemolysis (Mild to moderate hemolysis may cause false } \\
\text { increases in direct bilirubin and AST measurement. } \\
\text { Marked hemolysis can adversely affect all chemistry tests.) }\end{array}$} & \multicolumn{3}{|c|}{$\begin{array}{l}\text { Overall Model Test }-X^{2}=9.82 \\
\text { df } 3, p=.02 \text { Binary logistic } \\
\text { regression to predict } \\
\text { hemolysis. Wald } X 2 \text { statistic }\end{array}$} & $\begin{array}{l}\text { The occurrence of hemolysis varied with group and sex. } \\
\text { Female rats showed more hemolysis (only } 1 \text { male in 3- } \\
\text { month data showed slight hemolysis). Hemolysis oc- } \\
\text { curred at a slightly higher rate in the control group ( } 50 \% \\
\text { of the female rats) compared to the HP group ( } 37.5 \% \text { of } \\
\text { the female rats). }\end{array}$ \\
\hline & $\begin{array}{l}X^{2}= \\
0.00, \mathrm{df} \\
1, \\
p=.99\end{array}$ & $\begin{array}{l}X^{2}=0.0 \\
\text { df } 1 \\
p=1.00\end{array}$ & $\begin{array}{l}x^{2}=0.0 \\
\text { df } 1 \\
p=.99\end{array}$ & \\
\hline
\end{tabular}

NOTE - Total Protein: $p$ value could be rounded to $p=.05$; if we interpret it, Placebo > 2014 HP 
Table 6 One Month Study Blood Chemistry Control and 2014HP group by Sex ANOVA (bold - significant differences)

\begin{tabular}{|c|c|c|c|c|}
\hline Serum measure & $\begin{array}{l}\text { GROUP } \\
\text { main effect }\end{array}$ & $\begin{array}{l}\text { SEX main } \\
\text { effect }\end{array}$ & $\begin{array}{l}\text { GROUP X } \\
\text { SEX } \\
\text { interaction }\end{array}$ & $\begin{array}{l}\text { Special Notes *Report as } \\
p<.001\end{array}$ \\
\hline & $\begin{array}{l}\text { F, } p \text { value } \\
(\mathrm{df} 1,19)\end{array}$ & $\begin{array}{l}\text { F, } p \text { value } \\
(\mathrm{df} 1,19)\end{array}$ & $\begin{array}{l}F, p \text { value } \\
(\mathrm{df} 1,19)\end{array}$ & \\
\hline Glucose (mg/dL) & $6.27, .02$ & $5.47, .03$ & $0.89, .36$ & $\begin{array}{l}\text { Placebo > } 2014 \text { HP \& Females } \\
<\text { Males }\end{array}$ \\
\hline Urea Nitrogen (mg/dL) & $0.04, .84$ & $.12, .74$ & $2.03, .17$ & No significant interaction \\
\hline Creatinine (mg/dL) & $0.13, .73$ & $24.89,<.001$ & $0.29, .60$ & Females > Males \\
\hline Sodium (mEq/L) & $2.07, .17$ & $0.01, .93$ & $2.65, .12$ & No significant interaction \\
\hline Potassium (mEq/L) & $27.70,<.001$ & $25.14,<.001$ & $29.88,<.001$ & $\begin{array}{l}\text { differences between the } \\
\text { groups is different for females } \\
\text { and males. } \\
\text { Females: Placebo }>2014 \mathrm{HP} \\
\text { Males: Placebo < } 2014 \mathrm{HP}^{* *}\end{array}$ \\
\hline Chloride (mEq/L) & $0.07, .80$ & $7.98, .01$ & $0.03, .87$ & Females > Males \\
\hline Bicarbonate (mEq/L) & $0.90, .36$ & $18.27,<.001$ & $6.95, .02$ & $\begin{array}{l}\text { differences between the } \\
\text { groups is different for females } \\
\text { and males. } \\
\text { Females: Placebo < } 2014 \text { HP } \\
\text { Males: Placebo > } 2014 \mathrm{HP}\end{array}$ \\
\hline Anion Gap (mEq/L) & $18.69,<.001$ & $0.23, .64$ & $2.83, .11$ & Placebo < 2014 HP \\
\hline Albumin (g/dL) & $0.17, .69$ & $77.95,<.001$ & $0.51, .49$ & Females $>$ Males \\
\hline Total Protein (g/dL) & $1.42, .25$ & $74.89,<.001$ & $0.26, .62$ & Females > Males \\
\hline Globulin (g/dL) & $6.40, .02$ & $16.81, .001$ & $0.03, .87$ & $\begin{array}{l}\text { Placebo }>2014 \mathrm{HP}^{* *} \& \\
\text { Females }>\text { Males }\end{array}$ \\
\hline Calcium (mg/dL) & $0.11, .74$ & $45.25,<.001$ & $0.91, .35$ & Females > Males \\
\hline Phosphorus (mg/dL) & $9.82, .005$ & $14.87, .001$ & $0.61, .45$ & $\begin{array}{l}\text { Placebo < } 2014 \text { HP \& Females } \\
<\text { Males }\end{array}$ \\
\hline Cholesterol (mg/dL) & $0.02, .90$ & $11.89, .003$ & $0.08, .78$ & Females $<$ Males \\
\hline Total Bilirubin (mg/dL) & $66.58,<.001$ & $4.29, .052$ & $4.29, .052$ & Placebo < 2014 HP** \\
\hline $\operatorname{ALT}(U / L)$ & $0.65, .43$ & $21.51,<.001$ & $0.20, .66$ & Females $<$ Males \\
\hline ALP (U/L) & $1.04, .32$ & $8.09, .01$ & $3.00, .10$ & Females $<$ Males \\
\hline GGT (U/L) & \multicolumn{4}{|c|}{$\begin{array}{l}\text { All values were " }<3 \text { " - no analyses } \\
\text { conducted }\end{array}$} \\
\hline CK (U/L) & $4.36, .05$ & $6.75, .02$ & $8.28, .01$ & $\begin{array}{l}\text { differences between the } \\
\text { groups is different for females } \\
\text { and males. } \\
\text { Females: Placebo < } 2014 \mathrm{HP} \\
\text { Males: Placebo > } 2014 \mathrm{HP}\end{array}$ \\
\hline \multirow[t]{2}{*}{$\begin{array}{l}\text { Hemolysis (Mild to moderate hemolysis may cause false increases in direct } \\
\text { bilirubin and AST measurement. Marked hemolysis can adversely affect all } \\
\text { chemistry tests.) }\end{array}$} & \multicolumn{4}{|c|}{$\begin{array}{l}\text { Overall Model Test }-X^{2}=9.04, \mathrm{df} 3 \\
p=.03 \\
\text { Binary logistic regression to predict } \\
\text { hemolysis. Wald X2 statistic }\end{array}$} \\
\hline & $\begin{array}{l}X^{2}=0.11 \\
\text { df } 1, p=.74\end{array}$ & $\begin{array}{l}X^{2}=0.0, d f \\
1, p=.99\end{array}$ & $\begin{array}{l}X^{2}=0.0, \mathrm{df} \\
1, p=1.0\end{array}$ & \\
\hline
\end{tabular}

\#*HP group(s) was significantly $(p<.05)$ different from Control Group

related difference did occur, with females having higher serum CK than males. Several changes in electrolytes were observed, also sex-related. While there were many sex related effects observed in the blood chemistry panel, bilirubin was the only variable that differed between the control and treatment groups in both 1-month and 3-month studies.
We speculate that the elevated bilirubin may be due to some hemolysis in the samples and also to Heme oxygenase-1 (HO-1) catalyzed breakdown of heme. We have recently observed induction of HO-1 in rats treated with $H$. procumbens aqueous-ethanolic extracts [31], and others have also inferred $H$. procumbens affects $\mathrm{HO}-1$ expression [32]. 
Understanding sex-related differences of antiinflammatories and analgesics used to treat pain is a major research priority [33]. Analgesics most frequently used for musculoskeletal conditions, such as non-steroidal antiinflammatory drugs (NSAIDs) cause toxicities in liver, kidney, cardiovascular and gastrointestinal function [34-36]. A recent analysis of patients in a large multicenter randomized clinical trial of celecoxib, naproxen and ibuprofen users for one year, identified males as having higher risk than females for major toxicities in either of the cardiovascular, gastrointestinal, or renal systems [37]. Determining whether well-defined botanicals used for pain management have similar toxicities is critically important.

Past studies of $H$. procumbens products suggest the antiinflammatory and analgesic properties are due to inhibition of eicosanoid and nitric oxide (NO) biosynthesis, by altering expression and/or inhibition of COX and LOX and inducible nitric oxide synthase (iNOS), and to altered expression of pro- and anti-inflammatory cytokines and other mediators [2,38]. Studies of well-defined products such as employed here, can answer whether $H$. procumbens extracts will inhibit both cyclooxygenases $1 / 2$ (COX) and 5-lipoxygenase (LOX) expression and/or activities. Such dual COX/LOX inhibitors should be more efficacious with fewer side effects than current (single target) NSAIDs, and may reduce the use of opioid painkillers.

\section{Conclusions}

Consumption of 7-10x the human equivalent dose of several chemically defined $H$. procumbens aqueousethanolic extracts by female and male Sprague Dawley for one and three months resulted in no significant histopathology of liver heart, kidney, heart, lung or GI tract. However, analysis of blood chemistry indicated significant treatment group by sex interaction differences for sodium, potassium and phosphate of 1-month study rats when considering control and the 2014HP and 2016 Butanolic HP extracts. When considering just the control and 2014HP extract, potassium and CK were significant in group by sex interations. In the 3-month study of 2014 HP extract, phosphorus was the only group by sex outcome that was significantly affected. It appears that blood chemistry and signficant sex-related effects are caused by subtle chemical differences in the HP extracts.

\section{Supplementary information}

Supplementary information accompanies this paper at https://doi.org/10. 1186/s12906-019-2789-9.

Additional file $\mathbf{1}$ a. Independently analyzed additional outcome measures of 1-month study group by sex ANOVA. b. 1-Month Study Histopathology Analysis: Test of Group Differences - Control versus 2014 HP ( $n=23 ; 11$ females, 12 males). c. 3-Month Study Histopathology
Analysis: Test of Group Differences - Control versus 2014 HP $(n=28 ; 16$ females, 12 males)

\section{Abbreviations}

AAVLD: American Association of Veterinary Laboratory Diagnosticians; ALP: Alkaline Phosphatase; ALT: Alanice Transaminase; CK: Creatine Kinase; COX-2: Cyclooxygenase 2; CRP: C-reactive protein; Cys-LT: Cysteinyl leukotrienes; DC: Devils's Claw; HED: Human Equivalent Dose; HP: H. procumbens; NSAIDs: Non-Steriodal Anti-Inflammatory Drugs;

PGE2: Prostaglandin E2; VMDL: Veterinary Medical Diagnostic Laboratory

\section{Acknowledgements}

We thank Ms. Sydney Tyler for help with treatment of rats and sample collection; Dr. Kim Dae Young for histopathology analysis of tissue samples; Dr. Ravi Nistala for advice about the blood chemistry analyses; Dr. Zhentian Lei for HPLC-MS analyses; and the NIH for financial support.

\section{Authors' contributions}

$\mathrm{KJ}$, contributed to experimental design, treatment of rats, collection of samples and analyses, writing of manuscript. AP, contributed to the experimental design, collection of samples and interpretation of results. EAG$B$, contributed to collection of samples and analysis. MG, contributed to analysis of results and interpretation. WF, contributed to experimental design, analysis of extracts, analysis of data and writing of manuscript. All authors read and approved of the final version of the manuscript.

\section{Funding}

Financial support was provided by Grant R21AT009086 from the NIH/ National Center for Complementary and Integrative Health $(\mathrm{NCClH})$ and the University of Missouri. The contents are solely the responsibility of the authors and do not necessarily reflect the views of the sponsors. Sponsors approved the design of the study but had no role in the collection, analysis and interpretation of the results.

Availability of data and materials

Raw data generated is available at:

https://doi.org/10.32469/10355/67307

Ethics approval and consent to participate

Animal use was conducted by protocol (\#8654) approved by the University of Missouri Institutional Animal Care and Use Committee.

Consent for publication

Not applicable.

\section{Competing interests}

The authors declare that they have no competing interests.

\section{Author details}

${ }^{1}$ Department of Biochemistry, 117 Schweitzer Hall, University of Missouri-Columbia, Columbia, MO 65211, USA. ²Department of Medical Pharmacology and Physiology, MA 415 Medical Sciences Building, One Hospital Drive, Columbia, MO 65212, USA. ${ }^{3}$ Department of Biomedical and Health Informatics, University of Missouri-Kansas City, School of Medicine, M5-121, Kansas City, MO 64108-2792, USA.

Received: 19 January 2019 Accepted: 8 December 2019 Published online: 15 January 2020

References

1. Harpagophyti radix. 2016 [cited 2018 April 25]; Available from: http://www. ema.europa.eu/ema/index.jsp?curl=pages/medicines/herbal/medicines/ herbal_med_000113.jsp\&mid=WC0b01ac058001fa1d.

2. Mncwangi $\mathrm{N}$, et al. Devil's claw-a review of the ethnobotany, phytochemistry and biological activity of Harpagophytum procumbens. J Ethnopharmacol. 2012;143(3):755-71.

3. Mncwangi NP, et al. What the devil is in your phytomedicine? Exploring species substitution in Harpagophytum through chemometric modeling of 1H-NMR and UHPLC-MS datasets. Phytochemistry. 2014;106:104-15. 
4. Oltean $\mathrm{H}$, et al. Herbal medicine for low-back pain. Cochrane Database Syst Rev. 2014;12:CD004504.

5. Gagnier JJ, et al. Herbal medicine for low Back pain: a Cochrane review. Spine (Phila Pa 1976). 2016;41(2):116-33.

6. Marriott I, Huet-Hudson YM. Sexual dimorphism in innate immune responses to infectious organisms. Immunol Res. 2006:34(3):177-92.

7. Straub RH. The complex role of estrogens in inflammation. Endocr Rev. 2007;28(5):521-74.

8. Cutolo $\mathrm{M}$, et al. The immunomodulatory effects of estrogens: clinical relevance in immune-mediated rheumatic diseases. Ann N Y Acad Sci. 2010; 1193:36-42.

9. Blagojevic $M$, et al. Risk factors for onset of osteoarthritis of the knee in older adults: a systematic review and meta-analysis. Osteoarthr Cartil. 2010; 18(1):24-33.

10. Pereira $D$, et al. The effect of osteoarthritis definition on prevalence and incidence estimates: a systematic review. Osteoarthr Cartil. 2011;19(11): 1270-85.

11. Srikanth VK, et al. A meta-analysis of sex differences prevalence, incidence and severity of osteoarthritis. Osteoarthr Cartil. 2005;13(9):769-81.

12. Felson DT, Zhang Y. An update on the epidemiology of knee and hip osteoarthritis with a view to prevention. Arthritis Rheum. 1998;41(8):1343-55.

13. Felson DT, et al. The incidence and natural history of knee osteoarthritis in the elderly. The Framingham Osteoarthritis Study. Arthritis Rheum. 1995; 38(10):1500-5.

14. Lawrence RC, et al. Estimates of the prevalence of arthritis and selected musculoskeletal disorders in the United States. Arthritis Rheum. 1998;41(5): 778-99.

15. Schiphof D, et al. Factors for pain in patients with different grades of knee osteoarthritis. Arthritis Care Res (Hoboken). 2013;65(5):695-702.

16. Boyan $\mathrm{BD}$, et al. Hormonal modulation of connective tissue homeostasis and sex differences in risk for osteoarthritis of the knee. Biol Sex Differ. 2013; 4(1):3.

17. Karsdal MA, et al. The pathogenesis of osteoarthritis involves bone, cartilage and synovial inflammation: may estrogen be a magic bullet? Menopause Int. 2012;18(4):139-46.

18. Pfeilschifter J, et al. Changes in proinflammatory cytokine activity after menopause. Endocr Rev. 2002;23(1):90-119.

19. Razmjou S, et al. Effect of the menopausal transition and physical activity energy expenditure on inflammatory markers: a MONET group study. Menopause. 2016;23(12):1330-8.

20. Guo R, et al. Botany, Phytochemistry, pharmacology and toxicity of Strychnos nux-vomica L.: a review. Am J Chin Med. 2018;46(1):1-23.

21. Vlachojannis J, Roufogalis BD, Chrubasik S. Systematic review on the safety of Harpagophytum preparations for osteoarthritic and low back pain. Phytother Res. 2008;22(2):149-52.

22. Devil's claw root: ulcers and gastrointestinal bleeding? Prescrire Int. 2013; 22(144):296

23. Cuspidi C, et al. Systemic hypertension induced by Harpagophytum procumbens (devil's claw): a case report. J Clin Hypertens (Greenwich). 2015;17(11):908-10.

24. Posadzki P, Watson LK, Ernst E. Adverse effects of herbal medicines: an overview of systematic reviews. Clin Med (Lond). 2013;13(1):7-12.

25. Carvalho RR, et al. Syndrome of inappropriate antidiuretic hormone secretion induced by the phytotherapy Harpagophytum procumbers: case report. J Bras Nefrol. 2017;39(1):79-81.

26. Newall CA, Anderson LA, Phillipson JD. Herbal medicines : a guide for healthcare professionals. 1996. London: Pharmaceutical Press.

27. Shaw D, et al. Traditional remedies and food supplements. A 5-year toxicological study (1991-1995). Drug Saf. 1997;17(5):342-56.

28. Whitehouse LW, Znamirowska M, Paul CJ. Devil's claw (Harpagophytum procumbens): no evidence for anti-inflammatory activity in the treatment of arthritic disease. Can Med Assoc J. 1983;129(3):249-51.

29. Al-Harbi NO. Toxicity studies on Harpagophytum procumbens (Devil's claw) capsules in mice. J Med Plants Res. 2013;7(42):3089-97.

30. Davari SA, Miri A, Shahraki E. Teratogenic effects of Harpagophytum procumbens Ethanolic extract in mice and fetuses. Zahedan J Res Med Sci. 2016;18(10):e3481.

31. Ungerer, G.C., J; Ndam, ; Bekemeier, M; Song, H; Li, R; Siedhold, H; Yang, B; Appenteng, M; Greenlief, M, Miller D; Sun, G; Folk, W; Gu, Z, Harpagophytum procumbens extract ameliorates allodynia and modulates oxidative and antioxidant stress pathways in a rat model of spinal cord injury. Neuromolecular Medicine, 2020. In press.

32. Parenti $C$, et al. Involvement of the Heme-Oxygenase pathway in the Antiallodynic and Antihyperalgesic activity of Harpagophytum procumbens in rats. Molecules. 2015;20(9):16758-69.

33. in Relieving Pain in America: A Blueprint for Transforming Prevention, Care, Education, and Research. 2011: Washington (DC)

34. Kim S, Joo KW. Electrolyte and acid-base disturbances associated with nonsteroidal anti-inflammatory drugs. Electrolyte Blood Press. 2007;5(2):116-25.

35. Schmeltzer PA, et al. Liver injury from nonsteroidal anti-inflammatory drugs in the United States. Liver Int. 2016:36(4):603-9.

36. Knights KM, Mangoni AA, Miners JO. Non-selective nonsteroidal antiinflammatory drugs and cardiovascular events: is aldosterone the silent partner in crime? Br J Clin Pharmacol. 2006;61(6):738-40.

37. Solomon $\mathrm{DH}$, et al. Derivation and validation of a major toxicity risk score among nonsteroidal Antiinflammatory drug users based on data from a randomized controlled trial. Arthritis Rheumatol. 2019;71(8):1225-31.

38. Anauate MC, Torres LM, de Mello SB. Effect of isolated fractions of Harpagophytum procumbens D.C. (devil's claw) on COX-1, COX-2 activity and nitric oxide production on whole-blood assay. Phytother Res. 2010; 24(9):1365-9.

\section{Publisher's Note}

Springer Nature remains neutral with regard to jurisdictional claims in published maps and institutional affiliations.
Ready to submit your research? Choose BMC and benefit from:

- fast, convenient online submission

- thorough peer review by experienced researchers in your field

- rapid publication on acceptance

- support for research data, including large and complex data types

- gold Open Access which fosters wider collaboration and increased citations

- maximum visibility for your research: over $100 \mathrm{M}$ website views per year

At $\mathrm{BMC}$, research is always in progress.

Learn more biomedcentral.com/submissions 